\title{
Replanteando la cuestión del desarrollo y su relación dialéctica con la exportación de fuerza de trabajo de cara al siglo XXI
}

\author{
Reframing the development question and its dialectic \\ relationship with the export of labor force in the 21st Century
}

Raúl Delgado Wise*

ISSN IMPRESO 1870-7599 | ISSN RED CÓMPUTO 2448-7783 | 7-24

RECIBIDO 17/10/20 | ACEPTADO 2/12/20

\begin{abstract}
Resumen. Un elemento central del capitalismo e imperialismo contemporáneos es la emergencia de una nueva división internacional del trabajo que reconfigura las relaciones de poder y dependencia en el horizonte Norte-Sur o centro-periferia. Se trata de la exportación — directa e indirecta o incorpórea- de fuerza de trabajo. Tal proceso, comandado por el capital monopolista, adquiere su connotación más amplia con la exportación de fuerza de trabajo altamente calificada. El propósito de este artículo es profundizar en el análisis de ese fenómeno, tomando en consideración la profunda reestructuración que experimentan los sistemas de innovación en la actualidad, con Silicon Valley a la vanguardia. Es, en esencia, un fenómeno que trastoca las relaciones de dependencia entre países, desencadena nuevas y severas modalidades de intercambio desigual y da paso a un significativo replanteamiento de la cuestión del desarrollo de cara al siglo XXI y, particularmente, en tiempos de la covid-19.
\end{abstract}

Palabras clave: migración altamente calificada, exportación de fuerza de trabajo, ecosistemas de innovación, desarrollo de las fuerzas productivas, relaciones centro-periferia.

\begin{abstract}
A central element of contemporary capitalism and imperialism is the emergence of a new international division of labor that reshapes the power and dependence relations along the North-South or center-periphery axes. It involves the exportation - direct and indirect or incorporeal - of labor force. This process, driven by monopolistic capital, earns its broadest connotation with the export of highly qualified labor. The aim of this article is to deepen the analysis of that phenomenon, taking into consideration the deep restructuring that innovation systems are currently experiencing, with Silicon Valley leading the way. It is, essentially, a phenomenon that subverts the dependency relationship between countries, unleashing new and exacting modalities of unequal exchange and leads to a significant reframing of the development question in the 21st Century, and particularly within the era of covid-19.
\end{abstract}

Keywords: highly skilled migration, labor force export, innovation ecosystems, development of the productive forces, center-periphery relationships.

* Mexicano. Unidad Acádemica en Estudios del Desarrollo, Universidad Autónoma de Zacatecas, México. Correo-e: rdwise@uaz.edu.mx 


\section{Introducción}

La pandemia de la covid-19 se perfila como un punto de quiebre en la historia del capitalismo contemporáneo. Por su carácter disruptivo y el contexto en el que se despliega, signado por una profunda crisis sistémica — de dimensiones civilizatorias o epocales-, todo indica que habrá un antes y un después de la pandemia y que ese después apunta hacia el final de la llamada era neoliberal.

Ante este escenario, resulta importante analizar el significado e implicaciones de la llamada contrarrevolución neoliberal en América Latina y el Caribe. No podemos ignorar, en ese sentido, el amplio abanico de posturas que desde el pensamiento crítico latinoamericano se han enarbolado y que van desde el neo y postdesarrollismo, extractivismo y dependentismo, hasta concepciones descolonialistas y postcapitalistas vinculadas a la teoría y la práctica de los nuevos movimientos sociales (Veltmeyer y Delgado, 2018; Munck y Delgado, 2019). Por encima de los múltiples aportes que en tal perspectiva se han hecho al debate académico y político en la materia, hay tres aspectos críticos de la problemática que resultan estratégicos para comprender la encrucijada por la que atraviesan los países de la región: a) la redistribución geográfica de la producción mundial mediante el desplazamiento de partes de los procesos productivos industriales hacia la periferia del sistema en busca de fuerza de trabajo barata y flexible; b) la profunda reestructuración que experimentan los ecosistemas de innovación, con Silicon Valley a la vanguardia; y c) las nuevas dinámicas que acusa la migración contemporánea y que involucran una creciente participación de científicos y tecnólogos provenientes de países periféricos o emergentes.

Lo significativo de esos aspectos — sustentados en las posibilidades abiertas por la tercera y cuarta revoluciones científico-tecnológicas - es que a través de ellos se perfila una nueva división internacional del trabajo que acentúa las dinámicas de intercambio desigual y que redefine, en un sentido profundo, las relaciones de dependencia: la exportación de fuerza de trabajo, es decir, la exportación de la principal mercancía para la valorización del capital.

Partiendo de las consideraciones anteriores, el propósito del presente artículo es analizar este fenómeno poniendo el acento en la reestructuración de los sistemas de innovación y la migración calificada. Interesa, ante todo, dar cuenta del nuevo escenario, el cual nos convoca a replantear la cuestión del desarrollo de cara al siglo XXI. 


\section{Génesis de una nueva división internacional del trabajo}

Desde finales de la década de 1970, las grandes corporaciones multinacionales inician un proceso de reestructuración tendiente a trasladar parte de sus procesos productivos a zonas periféricas en busca de fuerza de trabajo barata y flexible. Se trata, en el fondo, de un nuevo «nomadismo» en el sistema de producción mundial sustentado en los enormes diferenciales salariales que existen y se reproducen en el horizonte Norte-Sur, i. e. el llamado arbitraje laboral global (Foster et al., 2011a:18). Ello ha dado lugar a la configuración de cadenas globales de valor, o mejor aún, redes globales de capital monopolista, mediante la instauración de plataformas de exportación que operan como economías de enclave en los países periféricos (Delgado y Martin, 2015). Este viraje estratégico en la organización de la producción industrial ha sido a todas luces espectacular: "Las 100 mayores corporaciones globales han desplazado su producción en forma más decisiva hacia sus filiales extranjeras [principalmente en el Sur], en que ahora se encuentran cerca de 60 por ciento del total de sus bienes y de sus empleados y más de 60 por ciento de sus ventas a nivel global» (UNCTAD, 2010). En similar tenor, se estima que en la periferia hay alrededor de 100 millones de trabajadores directamente empleados en plantas de ensamble establecidas en más de 5 mil 400 zonas de procesamiento que operan en al menos 147 países (UNCTAD, 2020). Esta situación ha transformado significativamente la geografía global de la producción, a grado tal que en la actualidad la mayor parte del empleo industrial (más de 70 por ciento) se localiza en países periféricos (Foster et al., 2011b).

Lo importante a destacar de dicho fenómeno es que no implica una industrialización de la periferia, sino que comprende un doble proceso regresivo que hemos conceptualizado como subprimarización económica. Eso significa que, lejos de transitar hacia un modelo de exportación manufacturera, lo que en realidad se exporta, bajo el disfraz de una exportación de bienes manufacturados, es fuerza de trabajo sin que ésta salga del país. No debe perderse de vista que las plantas de ensamble y empresas maquiladoras instaladas en países periféricos operan con insumos importados y regímenes de exención tributaria, lo que significa que la sustancia de lo que a través de ellas se exporta es la fuerza de trabajo incorporada al proceso productivo. De ahí que se trate de una exportación indirecta o incorpórea (disembodied) de fuerza de trabajo con el fetiche de una exportación de productos manufacturados (Cypher y Delgado, 2012; 
Márquez y Delgado, 2012). Tres consideraciones en relación con la génesis e implicaciones de esa peculiar modalidad exportadora resultan pertinentes.

La primera se refiere a la implantación de los programas de ajuste estructural, en tanto pilares de la reestructuración neoliberal cimentados en la tríada apertura, privatización y desregulación. El cometido de tales programas, impuestos por el Banco Mundial (BM) y el Fondo Monetario Internacional (FMI) fue -y continúa siendo - el desmantelamiento y desarticulación de los aparatos productivos de dichas economías para su rearticulación, asimétrica y subordinada, a las dinámicas de acumulación de las principales potencias imperialistas siguiendo la batuta del capital monopolista.

Como corolario o consecuencia de ese viraje trascendental, en la segunda consideración los mercados laborales se empequeñecen y precarizan, lo que propicia una desbordante masa de población redundante que es arrojada a las filas de la informalidad o forzada a emigrar en dirección sur-norte. La exportación directa de fuerza de trabajo, a través de la migración laboral, es sometida a graves condiciones de vulnerabilidad y limitaciones en sus derechos laborales y humanos. Tómese en consideración que, bajo la égida neoliberal, se liberaliza el comercio de todas las mercancías con excepción de la fuerza de trabajo, la cual es forzada a emigrar de sus países de origen y es expuesta a regímenes migratorios restrictivos que generan - como política de Estado - una creciente masa de población «ilegal» o indocumentada que, como ocurre en el caso de Estados Unidos, paga impuestos sin recibir beneficios sociales. Dicha fuerza de trabajo, tildada de «ilegal», pero indispensable para cubrir necesidades del mercado laboral, es sometida a condiciones de superexplotación laboral, discriminación y xenofobia; situación que además de ocultar las significativas contribuciones que los migrantes hacen a las economías y sociedades de destino, contribuye a criminalizarlos y convertirlos en enemigos públicos con importantes dividendos políticos para la ultraderecha y el neofascismo.

No se trata sólo del abaratamiento del costo de la fuerza laboral migrante, sino, en un sentido más amplio, de una modalidad de intercambio desigual entre países de origen y destino. Tómese en cuenta que la fuerza de trabajo que emigra no creció por generación espontánea ni se educó gratuitamente, sea cual sea su nivel de estudios. Sus costos de reproducción social y formación educativa corrieron a cargo de las familias del migrante y del fondo de capital social administrado por el Estado del país de origen. Esos costos, al compararlos con el acumulado de remesas enviadas a sus países de origen, tienden a ser bastante 
más onerosos. Ello implica que, a contrario sensu de lo que pregonan el BM y otras instituciones al servicio de los intereses de Estados Unidos y otras potencias imperialistas, las remesas - y por ende la migración laboral — no representan un subsidio Norte-Sur, sino exactamente lo opuesto: un subsidio Sur-Norte (Delgado y Gaspar, 2018).

La tercera consideración es que, además de la exportación directa de fuerza de trabajo, la exportación indirecta o incorpórea de la misma profundiza las relaciones de intercambio desigual entre países periféricos y centrales, en virtud de que lo que se transfiere al exterior es, por medio de las plantas de ensamble - en su mayoría reducidas a condiciones de comercio intrafirma o esquemas similares de compraventa de mercancías de exportación-, en esencia, la ganancia - en tanto forma transfigurada del plusvalor generado- al exterior. Constituye, por consiguiente, una modalidad de intercambio desigual que semeja, a escala nacional, el intercambio que se produce entre trabajo y capital en el proceso laboral. Es difícil imaginar una modalidad más lacerante de intercambio desigual entre países, con el agravante de que lo que se queda en el país de origen son salarios y prestaciones laborales muy inferiores a los que se otorgarían en el país de destino. Se gestan así los cimientos de una nueva división internacional del trabajo fincada en la exportación directa e indirecta o incorpórea de fuerza de trabajo que, en un primer momento, se nutre de mano de obra de baja o relativamente baja calificación.

\section{Reestructuración de los ecosistemas de innovación y exportación de fuerza de trabajo en sentido amplio}

La exportación de fuerza de trabajo — sea directa o indirecta (incorpórea) adquiere su connotación más amplia al incorporar fuerza de trabajo calificada y altamente calificada. Este paso, que implica el tránsito de una exportación de fuerza de trabajo en sentido restringido a otra en sentido amplio o lato, es un fenómeno relativamente reciente asociado a la profunda reestructuración que experimentan los ecosistemas de innovación en el siglo XXI.

Entonces, resulta vital en esta perspectiva penetrar en las características del ecosistema de innovación más avanzado en la actualidad: aquel hegemonizado por Estados Unidos y georreferenciado en Silicon Valley y que opera como una poderosa máquina de patentamiento con articulaciones en varios países periféricos 
y emergentes. La forma de organización del general intellect — concepto acuñado por Marx para enfatizar el carácter social del conocimiento acumulado-que se realiza en dicho complejo ecosistema permite poner a disposición de las grandes corporaciones multinacionales la capacidad científica y tecnológica de una impresionante y creciente masa de trabajadores calificados y altamente calificados provenientes o formados en diferentes países del mundo, tanto del centro como de la periferia del sistema. En la nueva trama entran en interacción numerosos agentes e instituciones que aceleran los ritmos de patentamiento y reducen los costos y riesgos asociados a la invención (Delgado, 2015; Delgado y Chávez, 2016; Míguez, 2013).

Algunos de los rasgos más sobresalientes de lo que se concibe como el Sistema Imperial de Innovación de Silicon Valley son:

1. La internacionalización y fragmentación de las actividades de investigación y desarrollo bajo modalidades «colectivas» de organización e impulso de los procesos de innovación: peer-to-peer, share economy, commons economy y crowdsourcing economy, mediante lo que se conoce como innovación abierta (open innovation). Se trata de modalidades de invención «extramuros», es decir, que se localizan fuera del entorno de la corporación multinacional y que entrañan la apertura y redistribución espacial de actividades intensivas en conocimiento, con la creciente participación de socios o agentes externos a las grandes corporaciones, tales como start-ups - empresas embrionarias de innovación-, proveedores de capital de riesgo, clientes, subcontratistas, head hunters, firmas de abogados, universidades y centros de investigación (Chesbrough, 2008). Esa nueva forma de organizar el general intellect da paso a una permanente configuración y reconfiguración de redes de innovación que interactúan en un complejo tejido interinstitucional comandado por el gran capital corporativo en mancuerna con el Estado imperial. Esta arquitectura en red trasciende, dinamiza y da complejidad a ritmos compulsivos, las formas precedentes de impulsar el cambio tecnológico.

Cabe destacar que, en ese entramado, el trabajo científico y tecnológico — desarrollado a través de agentes autónomos, las start-ups — no está subsumido formalmente al capital, en virtud de que los inventores no son empleados de las grandes corporaciones. De ahí que su subsunción sea sutil e indirecta, respaldada en un marco jurídico-institucional: el Tratado de Cooperación en Materia de Patentes (TCP), administrado por la Organización Mundial de la Propiedad Intelectual (OMPI), y un sofisticado tejido ecosistémico que propicia el desarrollo 
colectivo de los productos del general intellect a escala planetaria y su apropiación privada por la vía de las patentes y otra serie de mecanismos privativos, mediados por firmas de abogados al servicio de las grandes corporaciones multinacionales. Al respecto, se establece una dialéctica entre el conocimiento social acumulado, su impulso colectivo - acelerado por redes de científicos y tecnólogos-y su cercamiento y apropiación privada (Foladori, 2017).

2. La creación de ciudades científicas como Silicon Valley en los Estados Unidos y los nuevos «Silicon Valley» establecidos en los últimos años en áreas periféricas o regiones emergentes, principalmente en Asia — como es el caso de Bangalore en India-, donde se crean sinergias colectivas para acelerar los procesos de innovación (Bruche, 2009; Sturgeon, 2003). AnnaLee Saxenian (2006) lo describe como un nuevo paradigma georreferenciado, que se aparta de los viejos modelos de investigación y desarrollo y que abre el camino hacia una nueva cultura de la innovación basada en la flexibilidad, la descentralización y la incorporación, en diferentes modalidades, de nuevos y cada vez más numerosos jugadores que interactúan simultáneamente en espacios locales y transnacionales. Silicon Valley figura como el pivote de una nueva arquitectura de la innovación mundial, en torno al cual se tejen múltiples eslabones periféricos que operan como una suerte de maquiladoras científico-tecnológicas localizadas en regiones, ciudades y universidades alrededor del mundo. Ello da lugar a una nueva y perversa modalidad de intercambio desigual, a través de la cual los países periféricos y emergentes transfieren a los países centrales y al capital monopolista los costos de reproducción de la fuerza de trabajo altamente calificada involucrada en las dinámicas de innovación, así como el potencial para la generación de ganancias extraordinarias o rentas monopólicas de las innovaciones.

3. La implementación de nuevas formas de control y apropiación de los productos del trabajo científico-tecnológico por las grandes corporaciones multinacionales, a través de diversas formas de subcontratación, asociación, así como de manejo y diversificación de capital de riesgo. Dicho control se establece por una doble vía. De un lado, mediante equipos especializados de abogados al servicio de las grandes corporaciones, que conocen a fondo el marco institucional y las normas de operación de los sistemas de patentamiento. Dentro del complejo e intrincado marco jurídico-institucional impuesto por el TCP-OMPI resulta prácticamente imposible para un inventor independiente registrar y patentar, por sí solo, sus productos. De otro lado, existen bufetes de abogados que operan como cazadores de talento, contratistas, subcontratistas y gestores de diversa índole 
a favor de las grandes empresas asentadas en Silicon Valley. A esta nueva forma de injerencia y control corporativo de las dinámicas de innovación se le conoce como inversión estratégica (strategic investment) (Galama y James, 2008).

La manera como la gran corporación multinacional se inserta en esta dinámica -incubada y desplegada por medio del ecosistema de Silicon Valley y sus satélites - revela que, más que un agente propulsor del desarrollo de las fuerzas productivas sociales, el capital monopolista opera como un agente rentista, es decir, un agente que se apropia de los productos del general intellect sin participar en su gestación y desarrollo. En otras palabras, las ganancias extraordinarias que constituyen el leitmotiv del capital monopolista adquieren el carácter de rentas tecnológicas de acuerdo con el significado que Marx atribuye a la renta del suelo: la posibilidad de exigir una significativa porción del plusvalor social por el hecho de ser propietario de un bien, en este caso la patente, no producido ni reproducible por la fuerza de trabajo incorporada al proceso productivo. De ahí que en el capitalismo contemporáneo el capital monopolista haya dejado de fungir como un agente progresista, en tanto propulsor del desarrollo de las fuerzas productivas, y se torne en un ente parasitario, que incluso decide qué productos potencialmente trascendentes por su valor de uso ingresan al mercado y cuáles permanecen petrificados en la congeladora de la historia social (Foladori, 2017).

4. La expansión en el horizonte Norte-Sur de la fuerza de trabajo en áreas de ciencia, tecnología, ingeniería y matemáticas y el creciente reclutamiento de fuerza de trabajo altamente calificada proveniente de las periferias por mecanismos de outsourcing yoffshoring. Es importante consignar, en ese sentido, que la migración altamente calificada proveniente de los países periféricos desempeña un papel cada vez más relevante en los procesos de innovación, por lo que se forma una paradójica y contradictoria dependencia del Sur respecto del Norte: cada vez más los generadores de patentes son originarios de países periféricos y emergentes. Tal tendencia puede rastrearse en diferentes sectores de la economía global, incluidas la biotecnología agrícola y la biohegemonía en cultivos transgénicos, así como la apropiación del conocimiento indígena relacionado con tecnología de semillas (Gutiérrez y Fitting, 2016; Lapegna y Otero, 2016; Motta, 2016).

5. La creación de un marco institucional ad hoc orientado a la concentración y apropiación de los productos del general intellect a través de las patentes, bajo la tutela y supervisión de la OMPI en mancuerna con la Organización Mundial de Comercio (OMC) (Delgado y Chávez, 2016). Desde fines de la década de 1980 se advierte una tendencia a generar una legislación ad hoc en Estados Unidos, 
a tono con los intereses estratégicos de las grandes corporaciones multinacionales en materia de derechos de propiedad intelectual (Messitte, 2012). Mediante normas y reglamentaciones promovidas por la OMC, los alcances de dicha legislación se han ampliado significativamente. En esa perspectiva, la oficina del representante de comercio de Estados Unidos ha promovido la firma e implementación de Tratados de Libre Comercio (TLC). Debido a que por su naturaleza multilateral las disputas sobre propiedad intelectual dentro de la OMC tienden a ser cada vez más complejas, la estrategia de Estados Unidos incluye también negociaciones bilaterales de TLC como medidas complementarias para controlar los mercados e incrementar las ganancias corporativas. Los reglamentos establecidos por el TCP, modificados en 1984 y 2001 en el marco de la OMPI-OMC, han contribuido significativamente al fortalecimiento de esa tendencia.

\section{CUADRO 1}

Patentes solicitadas y otorgadas: total y 10 principales países, 1996-2018

\begin{tabular}{lrrrrrr}
\multicolumn{1}{c}{ Patentes } & Solicitadas & $\begin{array}{c}\text { Distribución } \\
\text { porcentual }\end{array}$ & Otorgadas & $\begin{array}{c}\text { Distribución } \\
\text { porcentual }\end{array}$ & $\begin{array}{c}\% \\
\text { otorgadas }\end{array}$ & Ranking \\
\hline Total & 45361224 & $100.0 \%$ & 19447764 & $100.0 \%$ & $42.9 \%$ & \\
Subtotal & 37412593 & $82.5 \%$ & 15696151 & $80.7 \%$ & $42.0 \%$ & 3 \\
China & 10497318 & $23.1 \%$ & 3138160 & $16.1 \%$ & $29.9 \%$ & 1 \\
Estados & 9862774 & $21.7 \%$ & 4646826 & $23.9 \%$ & $47.1 \%$ & 2 \\
Unidos & 8627834 & $19.0 \%$ & 4093992 & $21.1 \%$ & $47.5 \%$ & 2 \\
Japón & 3534255 & $7.8 \%$ & 1811789 & $9.3 \%$ & $51.3 \%$ & 4 \\
$\begin{array}{l}\text { República } \\
\text { de Corea }\end{array}$ & 1406340 & $3.1 \%$ & 357246 & $1.8 \%$ & $25.4 \%$ & 7 \\
$\begin{array}{l}\text { Alemania } \\
\text { Canadá }\end{array}$ & 842421 & $1.9 \%$ & 388204 & $2.0 \%$ & $46.1 \%$ & 6 \\
$\begin{array}{l}\text { Federación } \\
\text { Rusa }\end{array}$ & 831702 & $1.8 \%$ & 622539 & $3.2 \%$ & $74.9 \%$ & 5 \\
$\begin{array}{l}\text { India } \\
\text { Reino }\end{array}$ & 652043 & $1.4 \%$ & 130933 & $0.7 \%$ & $20.1 \%$ & 13 \\
Unido & 601246 & $1.3 \%$ & 165056 & $0.8 \%$ & $27.5 \%$ & 12 \\
Australia & 556660 & $1.2 \%$ & 341406 & $1.8 \%$ & $61.3 \%$ & 8 \\
\hline
\end{tabular}

Fuente: Sistema de Información sobre Migración y Desarrollo (SIMDE-UAZ), estimaciones con datos de World Intellectual Property Organization (WIPO), 1996-2018. 
Cabe resaltar que, de acuerdo con la naturaleza y características del Sistema Imperial de Innovación descrito, Estados Unidos figura como la potencia capitalista líder en innovación a escala mundial, al contabilizar 23.9 por ciento del monto total de solicitudes de patentes registradas en la OMPI entre 1996 y 2018; sin embargo, en el mismo lapso China superó a Estados Unidos en solicitudes de patentes: 23.1 por ciento versus 21.7 por ciento.

Es pertinente agregar que el dominio estratégico en materia de innovación ejercido por Estados Unidos a escala mundial se manifiesta no sólo por el volumen y ritmo de las patentes generadas, sino por el hecho de que:

a) 7 de las primeras 10 y 36 de 100 principales empresas innovadoras del mundo tienen su matriz en Estados Unidos (Thomson Reuters, 2018).

b) 46 de las 100 universidades más innovadoras del mundo se ubican en territorio estadounidense (Ewalt, 2018).

c) 7 de las 10 start-ups más exitosas del planeta se ubican en Estados Unidos (Murgich, 2015).

Más aún, conforme a la lógica extractiva/rentista que rige las nuevas dinámicas de innovación, la tasa de patentamiento de extranjeros en Estados Unidos se elevó de 18 por ciento en 1963 a 53.1 por ciento en 2018 (U.S. Patent and Trademark Office, 2019). Dicho incremento se ha visto favorecido por el papel que, en el ámbito de las políticas públicas, ha ejercido el gobierno de Estados Unidos para mantener, fortalecer y profundizar su liderazgo científico y tecnológico a escala planetaria. Esto, a su vez, guarda relación con la creciente demanda de fuerza de trabajo calificada de origen extranjero en Estados Unidos y otras potencias capitalistas en actividades intensivas en conocimiento. Esas actividades, como se aprecia en la gráfica 1, van más allá de la esfera de la innovación y se han convertido en el segmento más dinámico y estratégico de las principales potencias capitalistas, con Estados Unidos a la cabeza. ${ }^{1}$

${ }^{1}$ En las economías desarrolladas representan 35 por ciento del PIB y en Estados Unidos 39 por ciento (National Science Board, 2018). 


\section{GRÁFICA 1}

Estados Unidos: población inmigrante y nativa con estudios de nivel terciario en actividades intensivas en conocimiento por sector de actividad, 2018

\begin{tabular}{rlr} 
& \multicolumn{2}{c}{$\begin{array}{l}\text { Inmigrantes } \\
\text { Nativos }\end{array}$} \\
Cuidado de la salud & \multicolumn{1}{c}{$16.5 \%$} & $83.5 \%$ \\
Servicios financieros & $-13.9 \%$ & $86.1 \%$ \\
Servicios profesionales & $-14.2 \%$ & $85.8 \%$ \\
Tecnologías de la información & $-18.0 \%$ & $82.0 \%$ \\
Creación de conocimiento & $-12.7 \%$ & $87.3 \%$ \\
Servicios de alta tecnología & $-24.6 \%$ & $75.4 \%$ \\
Manufactura de alta tecnología & $28.5 \%$ & $71.5 \%$
\end{tabular}

Fuente: SIMDE-UAZ, estimación con base en U.S. Census Bureau, 2018; clasificación tomada de Hogan, 2011.

Además del impresionante respaldo en materia de inversión pública en ciencia básica y aplicada (equivalente a 2.74 por ciento del PIB en $2016^{2}$ ), el gobierno estadounidense se distingue sobre todo a partir de la década de 1990 por desplegar una agresiva política de atracción de talento externo promovida por la National Science Foundation, acompañada por un vigoroso aliento a una política migratoria altamente selectiva. En efecto, no es casual que la migración calificada y altamente calificada dirigida a ese país haya crecido a una tasa que poco más que duplica la correspondiente a la migración sin estudios superiores, como se aprecia claramente en la gráfica 2. 


\section{GRÁFICA 2}

Tasa de crecimiento anual (por cien) de la población de 22 años y más de edad inmigrante y nativa en Estados Unidos por nivel de escolaridad, 1990-2017

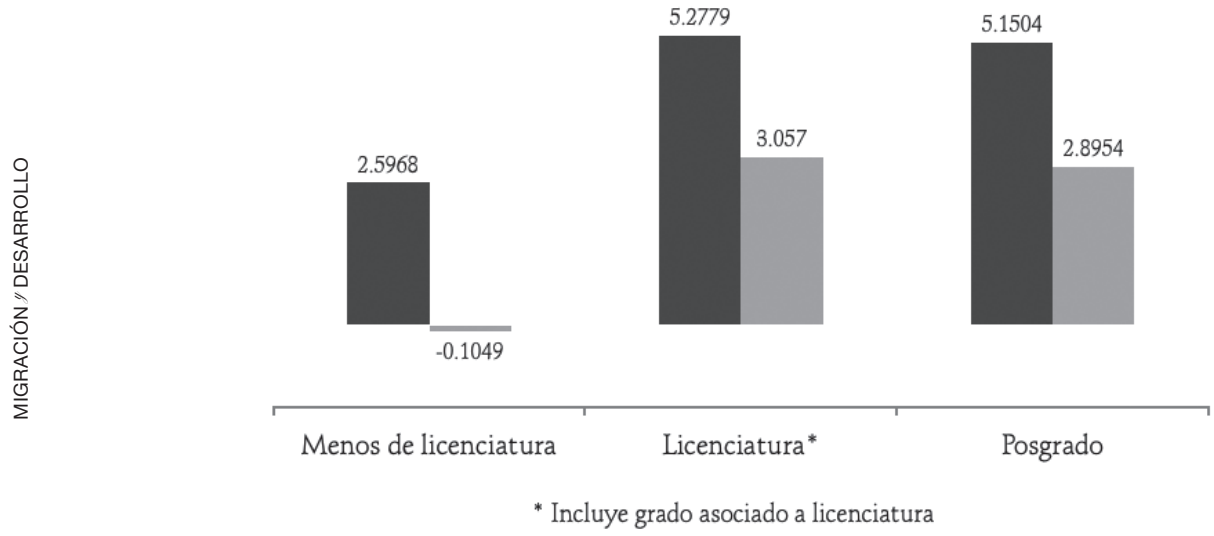

Fuente: SIMDE-UAZ, estimación con base en U.S. Census Bureau, 1990 y 2017.

Más aún, en la misma figura se aprecia que la participación de fuerza de trabajo calificada y altamente calificada proveniente del extranjero tiende a suplir y complementar el ritmo relativamente más pausado con el que crece la masa crítica de científicos y tecnólogos nacidos en Estados Unidos. No se trata sólo de una relación de complementariedad, sino de creciente dependencia de la capacidad de innovación de la fuerza de trabajo proveniente del extranjero. Otro dato significativo es que, como se aprecia en la gráfica 3, el segmento de inmigrantes calificados más dinámico es aquel que registra el más alto nivel de calificación.

Enmarcado en esta tendencia, otro rasgo revelador del nuevo perfil de la inmigración altamente calificada dirigida a Estados Unidos es que el grueso proviene de países periféricos o emergentes, como se desprende de la gráfica 4. En efecto, 8 de los 10 principales países que aportan inmigrantes con posgrado provienen de naciones emergentes. Y más todavía: el incremento se produce, como cabría esperarlo, principalmente con inmigrantes formados en áreas directamente vinculadas con la innovación: ciencia, tecnología, ingeniería y matemáticas (áreas CTIM). 


\section{GRÁFICA 3}

Tasa de crecimiento anual de la población inmigrante calificada en Estados Unidos
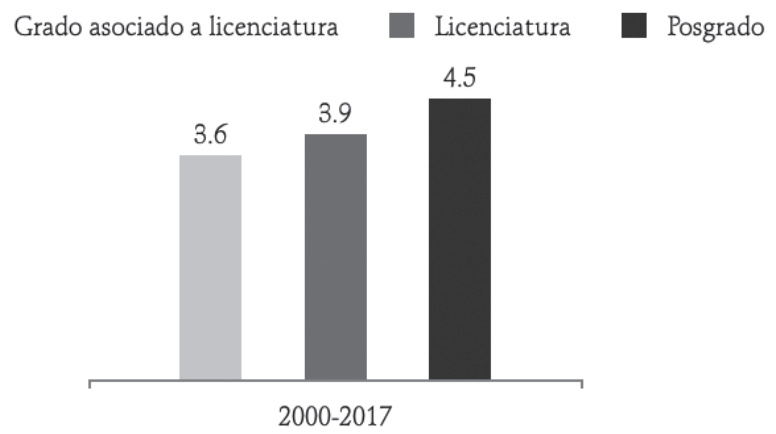

Fuente: SIMDE-UAZ, estimación con base en U.S. Census Bureau, varios años.

\section{GRÁFICA 4}

Inmigrantes con estudios de posgrados residentes en Estados Unidos

Tasa de crecimiento anual 1990-2017 (por cien)

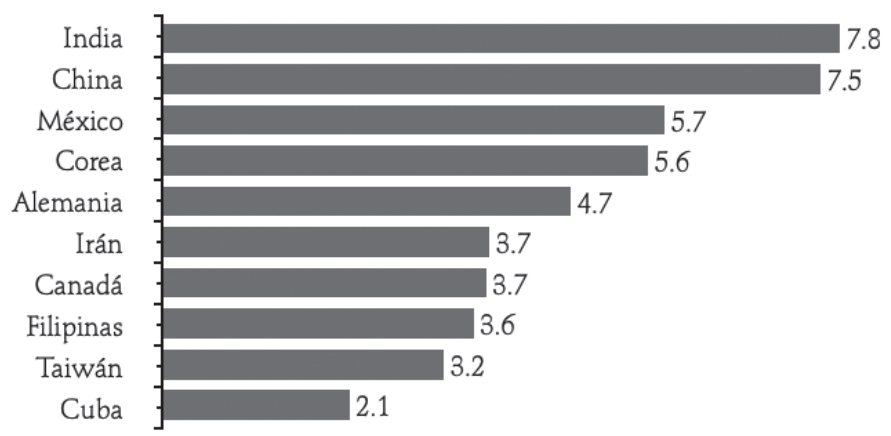

Fuente: SIMDE-UAZ, estimación con base en U.S. Census Bureau, 1990 y 2017.

Asimismo, existe una fuerte correlación entre los inmigrantes formados en áreas CTIM y el campo laboral en el que se desempeñan, en particular en ámbitos profesionales y en áreas asociadas con actividades de innovación. Queda claro, por tanto, que la reestructuración de los ecosistemas de innovación comandada por Estados Unidos ha dado lugar a una nueva migración calificada proveniente de países periféricos o emergentes, la cual está creciendo a un ritmo mayor que la migración en general y se nutre sobre todo por científicos y tecnólogos formados en áreas CTIM. 


\section{A manera de conclusión}

Con los avances del conocimiento y en concreto a raíz del advenimiento de las TIC y la llamada revolución de las tecnociencias, «el conocimiento y el cambio tecnológico [se sitúan] en el centro de los procesos de valorización del capital» (Míguez, 2013:27). Ante la innegable relevancia del conocimiento como propulsor de las dinámicas de acumulación de capital y la creciente producción de bienes intensivos en conocimiento, al capitalismo contemporáneo suele denominársele con el adjetivo de cognitivo. Empero, ello no significa que el móvil del sistema sea el conocimiento, más bien éste se convierte en un poderoso medio para acrecentar las ganancias y en específico las ganancias extraordinarias del capital monopolista. Así, la figura de la propiedad intelectual, existente desde hace siglos, emerge con más fuerza que nunca, pues permite objetivar el conocimiento, cercándolo como si fuese un derecho privativo. En opinión de Bolívar Echeverría, «la primera tarea que cumple la economía capitalista es la de reproducir la condición de existencia de su propia forma: construiry reconstruir incesantemente una escasez artificial, justo a partir de las posibilidades renovadas de la abundancia» (2011:85). La forma jurídica de la propiedad intelectual, en tanto derecho exclusivo sobre una invención a través de la figura de las patentes, permite la limitación, la parcelación del conocimiento, su mercantilización y su escasez artificial.

En ese contexto, el incremento de la migración internacional y su creciente selectividad no pueden ser comprendidos — como se ha argumentado-al margen de la dinámica y las contradicciones que encierra el capitalismo contemporáneo. De ahí que la nueva migración calificada y altamente calificada no pueda ser comprendida al margen de la profunda metamorfosis que experimenta el capital monopolista, tanto en la redistribución geográfica de las actividades manufactureras como en la reestructuración de los ecosistemas de innovación. Tal metamorfosis se sustenta en las posibilidades abiertas por la tercera y cuarta revoluciones industriales, al tiempo que da paso a la consolidación de una nueva división internacional del trabajo en el horizonte NorteSur: la exportación directa e indirecta de fuerza de trabajo, que adquiere su connotación más amplia con la inclusión del segmento de fuerza de trabajo calificada y altamente calificada. Esto, a su vez, propicia nuevas y extremas modalidades de intercambio desigual (Delgado y Martin, 2015).

Dada la importancia que en el desarrollo del general intellect tiene el trabajo intelectual (científico, tecnológico, inmaterial), el hecho de que un creciente 
contingente de trabajadores intelectuales provenga, precisamente, de países periféricos o emergentes, plantea una paradoja hasta hace poco inimaginable: la innovación, como motor del desarrollo de las fuerzas productivas, depende cada vez más de la participación de científicos y tecnólogos del Sur que están al servicio del Norte y en contra del Sur. Al proyectársele en el horizonte NorteSur, la paradoja refleja una potencial reversión, en el plano del trabajo científico y tecnológico, de las tradicionales relaciones de dependencia. Complementariamente, conduce a un replanteamiento de la cuestión del desarrollo dentro de una nueva trama entre progreso y rentismo circunscrita en la contradicción entre progreso y barbarie que encierra la modernidad capitalista.

Ante dicho escenario, la pandemia de la covid-19 adquiere especial relevancia. Por su magnitud y trascendencia, representa una encrucijada de dimensiones civilizatorias o epocales en la historia del capitalismo. Por un lado, como lo subraya Luis Arizmendi: «Stricto sensu, la pandemia por covid-19 es la expresión implacable pero particular de una crisis epidemiológica planetaria de orden mayor, que se ha puesto al descubierto para cambiarlo todo, y que pone en evidencia sus mayores peligros desde su interacción con las demás dimensiones constitutivas de la crisis epocal del capitalismo» (2020:19).

Por otro lado, además de incubar una tendencia neoautoritaria que conlleva la posible instauración de un estado de excepción mundializado, abre también, a contrario sensu, una ventana de oportunidad para la potencial transformación o reconfiguración del sistema capitalista. Si antes de su irrupción el neoliberalismo estaba herido de muerte, con la pandemia se rubrica su acta de defunción. Es innegable, al respecto, que nos encontramos en presencia de una fase del capitalismo en la que sus contradicciones se exacerban a un grado extremo y los intentos de darle aliento artificial resultan cada vez más infructuosos.

Los signos vitales del capitalismo en tiempos de la covid-19 están preñados de una estela de incertidumbre. En el nivel internacional, el escenario está signado por la enconada disputa interimperialista entre Estados Unidos y China, donde la ruta que seguirá la primera potencia capitalista del mundo frente al inminente declive de la administración de Trump no deja de ser una incógnita. No obstante, por encima de estas y otras incertidumbres, lo cierto es que la actual coyuntura incuba también posibilidades inéditas de transformación social, que se perfilan a través de la forma misma de enfrentar al virus y sus consecuencias. 


\section{Referencias}

Arizmendi, Luis (2020), "La crisis epidemiológica global en el marco de la crisis epocal del capitalismo", Migración y Desarrollo, 18(34), pp. 7-32.

Banco Mundial (2018), "Research and development expenditure (\% of GDP)», The World Bank, en https://data.worldbank.org/indicator/gb.xpd.rsdv.gd.zs.

Bruche, Gert (2009), "The emergence of China and India as new competitors in MNCs», Innovation Networks. Competition and Change, 13(3), pp. 199-213.

Chesbrough, Henry (2008), "Open innovation: a new paradigm for understanding industrial innovation", en H. Chesbrough, W. Vanhaverbeke y J. West (eds.), Open innovation: Researching a new paradigm, Oxford, Oxford University Press, pp. 1-14.

Cypher, James y Raúl Delgado Wise (2012), México a la deriva. Génesis, desempeño y crisis del Modelo Exportador de Fuerza de Trabajo, México, Miguel Ángel Porrúa.

Delgado Wise, Raúl (2015), «Unraveling Mexican highly-skilled migration in the context of neoliberal globalization", en S. Castles, M. Arias Cubas y D. Ozkul (eds.), Social transformation and migration: national and local experiences in South Korea, Turkey, México and Australia, Basingstoke, Palgrave Macmillan, pp. 201-218.

Delgado Wise, Raúl y David Martin (2015), «The political economy of global labor arbitrage», en Kees van der Pijl (ed.), The international political economy of production, Cheltenham, Edward Elgar, pp. 59-75.

Delgado Wise, Raúl y Mónica Chávez (2016), «iPatentad, patentad!: apuntes sobre la apropiación del trabajo científico por las grandes corporaciones multinacionales», Observatorio del Desarrollo. Investigación, reflexión y análisis, 4(15), pp. 22-30.

Delgado Wise, Raúl y Selene Gaspar (2018), «Claves para descifrar la arquitectura de la globalización neoliberal: exportación de fuerza trabajo e intercambio desigual», en José Luis Calva (ed.), La globalización neoliberal en crisis, México, Juan Pablos Editor, pp. 159-186.

Ewalt, David M. (2018), «Reuters top 100: the world's most innovative universities 2018», en https://www.reuters.com/article/us-amers-reuters-ranking-innovative-univ/ reuters-top-100-the-worlds-most-innovative-universities-2018-idUSKCN1MLOAZ.

Foladori, Guillermo (2017), "Teoría del valor y ciencia en el capitalismo contemporáneo», Observatorio del Desarrollo. Investigación, reflexión y análisis, 6(18), pp. 42-47.

Foster, John Bellamy, Robert W. McChesney y Jamil Jonna (2011a), «The internationalization of monopoly capital», Monthly Review, 63(2), pp. 3-18.

(2011b), "The global reserve army of labour and the new imperialism, Monthly Review, 63(6), pp. 1-15. 
Galama, Titus y Hosek James (2008), US competitiveness in science and technology, Santa Mónica: RAND Corporation.

Gutiérrez Escobar, L. y E. Fitting (2016), "Red de Semillas Libres: crítica a la biohegemonía en Colombia», Estudios Críticos del Desarrollo, 7(11), pp. 85-106.

Hogan, Timothy (2011), "An overview of the knowledge economy, with a focus on Arizona", A report from the Productivity and Prosperity Project (P3), an initiative supported by the Office of the University Economist, Arizona State University, en https://wpcarey.asu.edu/sites/default/files/uploads/research/competitiveness -prosperity-research/Knowledge-Economy.pdf

Lapegna, Pablo y Gerardo Otero (2016), "Cultivos transgénicos en América Latina: expropiación, valor negativo y Estado», Estudios Críticos del Desarrollo, 6(11), pp. 19-44.

Márquez, Humberto y Raúl Delgado Wise (2012), Desarrollo desigual y migración forzada. Una mirada desde el Sur global, México, Miguel Ángel Porrúa.

Messitte, Peter (2012), «Desarrollo del derecho de patentes estadounidense en el siglo XXI. Implicaciones para la industria farmacéutica», en Arturo Oropeza y Víctor Manuel Guízar López (eds.), Los retos de la industria farmacéutica en el siglo XXI. Una visión comparada sobre su régimen de propiedad intelectual, México, Universidad Nacional Autónoma de México/Comisión Federal para la Protección contra Riesgos Sanitarios, pp. 179-200.

Míguez, Pablo (2013), "Del general intellect a las tesis del capitalismo cognitivo: aportes para el estudio del capitalismo del siglo XXI», Bajo el Volcán, 13(21), pp. 27-59.

Motta, Renata (2016), "Capitalismo global y Estado nacional en las luchas de los cultivos transgénicos en Brasil», Estudios Críticos del Desarrollo, 6(11), pp. 65-84.

Munck, Ronaldo y Raúl Delgado Wise (eds.) (2019), Reframing Latin American development, Abingdon, Routledge.

Murgich, V. (2015), "Las start-up más exitosas (y famosas) del mundo», Merca2.o, en https://www.merca20.com/las-startup-mas-exitosas-y-famosas-del-mundo.

National Science Board (2018), «Science \& Engineering Indicators 2018», en https:// www.nsf.gov/statistics/2018/nsb20181/figures.

Saxenian, AnnaLee (2006), The new argonauts. Regional advantage in a global economy, Boston, Harvard University Press.

Sturgeon, Timothy J. (2003), «What really goes on in Silicon Valley? Spatial clustering and dispersal in modular production networks», Journal of Economic Geography, 3(2), pp. 199-225. 
Thomson Reuters (2018), «The top 100 global technology leaders», en https://www. thomsonreuters.com/content/dam/ewpm/documents/thomsonreuters/en/pdf/ reports/thomson-reuters-top-100-global- tech-leaders-report.pdf.

UNCTAD (2010), World Investment Report 2010, Nueva York, United Nations.

(2020), «Special economic zones and urbanization», en https://unhabitat .org/special-economic-zones-sezs-and-urbanization.

U.S. Census Bureau (1990), «Samples Census 1990», en https://www.census.gov/ main/www/cen1990.html

(2017), «American Community Survey (ACS)», en https://www.census. gov/acs/www/data/data-tables-and-tools/data-profiles/2017/

(2018), «American Community Survey (ACS)», en https://www.census. gov/acs/www/data/data-tables-and-tools/data-profiles/2018/

Veltmeyer, Henry y Raúl Delgado Wise (2018), Critical development studies. An introduction, Halifax, Fernwood Publishing. 